\title{
UNA MIRADA CRÍTICA A LA ENSEÑANZA A DISTANCIA (SEGUNDA PARTE)
}

José Manuel Carrión Arias

Doctorando en la Universidad Nacional de Educación a Distancia (UNED), España

\section{INTRODUCCIÓN}

En el presente apartado se analizan los principales aspectos negativos que, a juicio del autor, presenta la EAD. El tema se ha articulado en cuatro áreas:

- El alumno.

- La metodología de las investigaciones realizadas.

- El papel de las TIC.

- Los estudios económicos.

\section{EL ALUMNO Y LAS CAUSAS DEL ABANDONO}

\section{Introducción. Un caso cercano}

Uno de los aspectos más conflictivos que la EAD presenta es el alto porcentaje de alumnos que abandonan esta modalidad de enseñanza. Tomando el caso de la UNED (García Aretio, 1994) por ejemplo, de una entrada global de 102.300 matriculados, a partir del año que comenzó su actividad, ocho más tarde, en 1981 se había dado un abandono del 72\% (73.656 alumnos) y sólo el 1\% de licenciados (1.023 alumnos).

Las consecuencias afectan no sólo al alumnado, sino a la propia Institución. Para el alumno adulto, la renuncia a seguir estudiando supone la frustración de las expectativas que tenía al emprender el proceso de aprendizaje y puede ocasionarle sentimientos de fracaso personal. En cuanto a la Institución, es obvio que los índices de deserción estudiantil son el principal exponente de su grado de eficacia cualitativa y económicamente.

Sin duda, tras la decisión de dejar los estudios no hay una sola causa, sino varias. El abandono es un fenómeno complejo que obedece a múltiples factores de diversa naturaleza; siempre habrá una razón que provoque el desenlace, pero se tratará de la gota que colme el vaso de una crisis previa en la que están presentes otras causas.

Antes de abordar estas razones, es interesante saber qué se entiende por abandono. Aunque es lógico plantearse cuál es el fenómeno a analizar, las instituciones van a utilizar en su beneficio la incorporación de este concepto a la hora de dar cuenta de sus cifras. Los criterios adoptados son:

- Se define como abandono sin comenzar (non-starters) el de aquellos estudiantes que no se han examinado nunca a pesar de haber estado matriculados uno o varios años. 
- Abandono real (drop-out) sería el de aquellos alumnos que llegan a examinarse antes de dejar la carrera, habiendo obtenido una o varias calificaciones en el período en que estuvieron matriculados.

El abandono global resultaría de la suma de los dos. Por otra parte, algunas instituciones aplican procedimientos de matrícula peculiares, que les permiten enmascarar sensiblemente los niveles de deserción. Es el caso de la UK Open University: aplica un procedimiento de matrícula provisional para los nuevos estudiantes, que disponen de tres meses de plazo para hacerla efectiva; las tasas de abandono se calculan a partir de la matrícula definitiva.

Siguiendo con el ejemplo de la UNED ${ }^{1}$, el volumen estimado para el primer tipo de abandono, desde 1973 hasta 1981, fue del 58\%. De esta manera quedarían en abandono real un 14\% (fig. 1); es decir, sólo uno de cada cinco abandonos es real. Durar en la UNED más de un año en los que no comienzan es la excepción; un 86\% abandonan después del primer año.

Por referir otro ejemplo de España, aunque no de enseñanza superior, en el caso del Centro Nacional de Educación Básica a Distancia (CENEBAD), el abandono sin comienzo fue del $25 \%$, frente a un $47 \%$ para el caso del real'.

\section{La situación en otros paises ${ }^{3}$}

En la Athabasca University, de Canadá, los fracasos se dan en alta proporción: si se cuenta el total de los matriculados, los cursos son superados en un porcentaje que oscila entre el 35 y el 40 por ciento, pero si se restan los que sólo se inscribieron sin llegar realmente a comenzar actividad académica alguna, la cifra se eleva al $65 \%$, aunque existen serias diferencias de unas carreras a otras.

En la Sukhothai Thammathirat Open University (STOU), de Thailandia, las causas de los abandonos se han estudiado mediante indicadores distintos. En el primer semestre de curso se llegó a un tercio de abandono de los inscritos; dos años más tarde, se descendió a un cuarto. El seguimiento del grupo que entró en 1981 probó que después de cinco meses quedaba un 30\% de supervivientes. Este índice de deserciones varía, lógicamente, de acuerdo con la duración del programa.

En 1983, el Instituto de Tecnología Educativa, órgano responsable de la investigación en la Open University, llevó a cabo un estudio sobre la deserción en esta Universidad (Woodley y Parlett, 1983). Se pudo constatar que:

- $\quad$ El $30 \%$ de los nuevos estudiantes no llegaron a formalizar su matrícula definitiva.

- El $25 \%$ de los finalmente inscritos, no llegaron a realizar la prueba final.

- $\quad$ El $5,9 \%$ de los que se presentaron al examen final no ganaron ningún crédito.

- El $30 \%$ los estudiantes finalmente matriculados no ganaron crédito de curso.

\footnotetext{
${ }^{1}$ García Aretio, L. (1994), op cit., p. 153.

${ }^{2}$ Ibidem, pp. 155-156

${ }^{3}$ Ibídem, pp. 146-148
} 


\section{Las causas}

En la figura 2 se recogen los porcentajes procesados tras preguntar a los profesores-tutores de la UNED a nivel nacional sobre cuáles eran los aspectos que consideraban más críticos a la hora de determinar las causas en el abandono de los estudios (Martínez, 1986).

A partir de los datos anteriores y del estudio de otros cuestionarios, se propusieron por orden de importancia las doce causas fundamentales del abandono de los estudios en el caso de la UNED (García Aretio, 1987):

- Atención a obligaciones laborales.

- Atención a la familia.

- Falta de dedicación al estudio.

- Falta de motivación.

- Imagen equivocada que se tiene sobre la institución.

- Dificultad de superar las pruebas presenciales.

- Falta de hábitos y técnicas de estudio.

- Inasistencia a las horas de tutoría.

- Ausencia de servicio de orientación durante los estudios.

- Distancia desde el domicilio al Centro Asociado.

- Deficiente formación básica.

- Dificultades de aprendizaje para asimilar el material.

En el caso del CENEBAD, los alumnos atribuían su abandono, en mayor medida, a causas psicosociales $(60 \%)$ que a causas relativas al sistema (35\%). La causa más señaladas en la decisión de abandonar sus estudios es la falta de tiempo para estudiar y en segundo lugar, la del esfuerzo que les supone la comprensión de algunas materias, o las razones de atención a la familia. Aunque con muy bajos porcentajes, entre las causas relativas al sistema, destacan: la lejanía del tutor, la escasa calidad del material de estudio o las dificultades de las pruebas de evaluación.

\section{ESTUDIOS SOBRE LA EAD}

\section{Cuestionando su fiabilidad}

La EAD es sin duda una de las bazas con la que pueden jugar los gobiernos en sus políticas educativas; hay interés en presentarla, al menos, como un producto de calidad. A pesar del gran volumen de material escrito, los planteamientos de muchos análisis y estudios sobre EAD tienen carencias en el diseño de sus investigaciones, ya que a menudo se basan:

- En los resultados de encuestas realizadas a estudiantes mediante un test.

- Estudiantes, claro está, de EAD. 
- Estudiantes, muchas veces, con una actitud satisfactoria hacia la EAD.

Muchos de estos estudios concluyen en una visión positiva de este tipo de enseñanza; pero el análisis parte viciado de raíz. Como pretende mostrar este apartado, la calidad general de esta clase de investigaciones puede ser cuestionada.

\section{Los defectos de las investigaciones ${ }^{4}$.}

No es infrecuente la ausencia de control para evitar variables extrañas. Buena parte de los estudios se basan principalmente en la comparación de resultados entre un grupo de control y otro experimental. Intentan medir la causa, esto es, el impacto de una tecnología específica sobre los resultados del aprendizaje, o el influjo sobre las actitudes, a partir del efecto, esto es, los estudiantes. Para valorar con precisión esta relación, otras causas potenciales no deberían poder influir en los resultados medidos.

La mayoría de los estudios no utilizan sujetos seleccionados de forma aleatoria. La forma más simple de evitar el influjo de variables extrañas es asignar los estudiantes aleatoriamente tanto a los grupos experimentales, como a los de control.

Los instrumentos de medición —cuestionarios, test, escalas de actitud, etc. - son cuestionables: ¿miden lo que se supone que deberían? Habitualmente en los estudios no aparece ninguna información sobre la validez y fiabilidad de estos instrumentos.

Ausencia de control en los efectos reactivos (GAY y AIRASIAN, 2000). La investigación educacional denomina de este modo a un número de factores asociados con el modo en que se conduce un estudio y las actitudes de los estudiantes. Un efecto reactivo es el denominado Novedad, y se refiere al incremento del interés, la motivación o la participación del estudiante por el hecho de estar haciendo algo novedoso, sin que deba tratarse de algo necesariamente mejor en sí. Otro efecto reactivo es el que se conoce como John Henry, consistente en la infravaloración, por parte de los grupos de control o sus profesores, quienes se sienten amenazados ante un competidor que les puede desbancar.

De modo no infrecuente se pone el énfasis en cursos individuales en lugar de programa académicos; se toma el todo por una parte.

La investigación en ocasiones no tiene en cuenta las diferencias entre estudiantes. Una parte importante de la investigación a distancia ha conducido a pensar que no hay diferencias significantes entre los niveles de logros de los grupos de EAD y los de enseñanza presencial. Sin embargo, hay una amplia varianza de logros y actitudes dentro de los grupos, indicadores de diferencias entre los discentes: edad, experiencia educacional o motivación. El estudiante típico, fruto del promedio de la muestra, es un espejismo que enmascara la variabilidad de la población. Lo que interesa es ver cómo aprende la persona y no los grupos.

Las investigaciones se focalizan mayoritariamente en el impacto de tecnologías individuales, más que en la interacción de múltiples tecnologías.

\footnotetext{
${ }^{4}$ The Institue for Higher Education Policy (1999): What's the difference? A review for contemporary research on the effectivenessof distance learning in Higher Education, informe preparado por la America Federation of Teacher y la National Education Association, pp. 1-7.
} 


\section{LAS TIC: ¿LUCES DE BENGALA?}

\section{¿Las TIC aumentan la calidad de la enseñanza?}

No todos los diseños de EAD tienen que basarse en el uso de internet, pero sin duda, los desarrollos actuales están abiertos e intentan incorporar las posibilidades que ofrecen las TIC (fig. 3). Es cuestión de imagen, pero lo importante es evaluar la calidad que aportan y si realmente se mejora.

Cuando se descubrieron las posibilidades educativas de la radio, la televisión, el audio y el vídeo se pensó que la educación cambiaría radicalmente: las clases podrían emitirse por estos medios, o enviarse masivamente a las aulas mediante cintas magnéticas de audio o vídeo. Evidentemente, no fue así. Las posibilidades de difusión de la información y el conocimiento crecieron sobremanera con estos medios, pero ¿fue igual el aprovechamiento para los procesos de enseñanza-aprendizaje?

No es infrecuente que muchos de los diseños multimedia para EAD caigan en el error de reproducir en la pantalla del ordenador el mismo texto que se encuentra en un manual impreso, el mismo mapa, igual dibujo, sin considerar para su diseño las normas más elementales de lecturabilidad: hay que acabar imprimiendo esas páginas. Además, en la cultura del libro, el desarrollo del discurso es siempre lineal , pues hay una consecutio, donde los medios preceden a los fines y el instrumento va antes que el producto-, en el caso del hipertexto no hay consecutio alguna (Sartori, 2002): no hay orden; se consigue un aprendizaje fragmentario carente de coordenadas generales y sin trabajo de síntesis. Las numerosas referencias cruzadas fomentan la desorientación.

De las investigaciones ${ }^{5}$ que se han realizado en torno a la incidencia de los diversos medios instructivos en el aprendizaje, como han puesto de manifiesto Clark (1983. 1994) y Kozma (1991, 1994), y con anterioridad Jamison y McAnany (1978), no se desprende una visión demasiado optimista en lo que a internet se refiere. Como ha señalado García Aretio (2001) no se ha encontrado un resultado contundente en cuanto a la mejora sustancial del aprendizaje cuando se utiliza un medio u otro; más que el medio, es el diseño instructivo. No se trata de que se apliquen unas u otras tecnologías más o menos avanzadas o sofisticadas, se trata de que esos medios o recursos se pongan al servicio de un determinado modelo de enseñanza-aprendizaje, tanto en su planificación o diseño, como en su desarrollo o aplicación y evaluación.

\section{TIC: ¿formación para todos?}

Se insiste mucho en que la democratización del acceso a la formación gracias a la EAD. Como en la Antigua Grecia, en el siglo $V$ a.C., cuando Atenas pasa de ser un régimen aristocrático a un estado democrático, todos los ciudadanos tenían acceso a los cargos públicos; todos podían aspirar a todo. ¿Es esto lo que permite, por ejemplo, el uso de internet? La brecha digital entre inforricos e infopobres está servida (fig. 4). Por otra parte, con cierta frecuencia internet sigue siendo lento, con colapsos que hacen que www se convierta en sinónimo de world wide wait, como se ha señalado (Ruipérez, 2003) con gran agudeza.

Al tener que hacerse mediante mensajes escritos la mayor parte de la comunicación entre estudiantes y profesor, y de aquéllos entre sí, se están exigiendo habilidades de escritura superiores a las

\footnotetext{
Educación.

${ }^{5}$ Los siguientes autores que se mencionan son citados por García Aretio (2001) en La educación a distancia, Ariel
} 
exigidas en la enseñanza cara a cara. Los individuos con escasas capacidades para este modo de comunicación pueden encontrarse en desventaja con aquellos otros más cualificados.

Una EAD basada en las TIC requiere unos ciertos niveles de alfabetismo informático, que serán posiblemente un obstáculo insalvable para algunos; el manejo de un procesador de textos, una hoja de cálculo, una base de datos, la instalación de software, la navegación y búsqueda de recursos en internet, o el uso del e-mail, son algunas de las destrezas que deben estar presentes en alguien que debe recurrir a las TIC para su estudio a distancia.

Un último aspecto democratizador es que, aunque en los últimos años han crecido los contenidos en español, internet habla mayoritariamente en inglés.

\section{TIC y capitalismo cultural}

Siguiendo con la Historia Clásica, con la democracia ateniense surgen entonces los sofistas: hombres capaces de enseñar a los ciudadanos a discutir con energía y precisión. La enseñanza, hasta entonces libre y desinteresada dedicación, pasa a convertirse en actividad mercantil: los sofistas realmente enseñaban por cobrar.

La cultura actualmente es un fenómeno inserto en el entramado industrial del sistema económico occidental: «La cultura se ha convertido simplemente en un pretexto para el progreso sociopolítico y el crecimiento económico" (Yudice, 2002). Cabe hablar de un capitalismo cultural en el que las TIC juegan un papel fundamental. La telefonía móvil, internet, los pdas, las cámaras digitales permiten transformar la experiencia empírica de las manifestaciones culturales como la música, la expresión icónica o la comunicación interpersonal, en una representación digitalizada en un soporte informático, para así ser distribuida a gran escala, sin limitaciones temporales ni espaciales.

La educación en general, y la formación en particular, están siendo sometidas, cada vez en mayor grado, a las presiones de la economía del mercado y de la ideología neoliberal que la acompaña. La actividad educativa, de forma similar a lo que está sucediendo con otras manifestaciones de la cultura humana, se ha convertido en una mercancía más; cada vez existe una mayor oferta de servicios educativos por los que hay que pagar para acceder a los mismos. Las empresas dedicadas al negocio de la formación son uno de los ámbitos en crecimiento del sector terciario de la economía: la oferta de cursos para la formación dentro de la empresa, las importantes inversiones y subvenciones movilizadas por las organizaciones empresariales y sindicales en el ámbito de la formación continua y ocupacional, la comercialización de cursos a distancia, el incremento de la oferta de postgrados universitarios,... son algunos de los fenómenos que configuran el proceso de mercantilización de la actividad educativa (Area, 2004).

En este sentido, las TIC están contribuyendo a la cosificación de la educación. Estas tecnologías permiten enlatar un curso, convirtiéndolo en un producto tangible que se puede ofrecer al público para su comercio y consumo. Los CD-ROM o DVD, así como la formación on-line o e-learning distribuida vía Internet, son los nuevos vehículos que permiten producir y difundir a gran escala cursos formativos en conserva. Por otra, las empresas tradicionalmente vinculadas con la producción de materiales curriculares impresos han comenzado a invertir en la creación de multimedia educativo. Se alcanza, entonces, el horizonte económico. 
5. ¿ECONOMÍAS DE ESCALA? SÍ, PERO...

\section{Introducción}

El debate sobre las implicaciones pedagógicas del uso de TIC en la educación está aumentando y produciendo un gran volumen de informes en los que se evalúa su efectividad en el aprendizaje, las actitudes de profesores y estudiantes, los diseños instruccionales y diversas modalidades educativas apoyadas en la tecnología. Sin embargo, ha sido poca la investigación sistemática realizada en cuanto a implicaciones financieras. Las instituciones continúan preocupándose por los aspectos clave de planificación e infraestructura de las TIC, tales como desarrollar un plan estratégico y financiero, planear la integración del currículo y brindar soporte adecuado al usuario. Se sabe que la introducción de la tecnología es costosa, aunque el problema de cómo calcular los costes queda en gran parte sin resolver.

Aunque hasta la fecha no hay evidencia de que la tecnología representará un ahorro generalizado en la educación superior, es necesario evaluar los costes en que se incurre por inversión en TIC a fin de establecer unas tasas de coste-beneficio. La pregunta clave es ¿cómo puede la tecnología mejorar la educación manteniendo un equilibrio en la relación coste-beneficio?, y no simplemente si es efectiva la tecnología o si puede la tecnología reducir los costes, lo cual equivale a identificar los escenarios y las condiciones educativas adecuadas en las cuales se pueda lograr un equilibrio entre los costes y los beneficios, justificando así la inversión.

\section{Los costes y el aprendizaje electrónico}

Los rápidos cambios que sufre la tecnología son una de las razones más obvias que dificultan la estimación de costes y la toma de decisiones de inversión. Sin embargo, hay muchos otros desafíos que se deben considerar para llegar a entender los costes de la TIC y poderlos comparar entre diversas instituciones de educación superior. Entre los factores compuestos están los siguientes (Claeys, 2004):

- Factores motivados externamente:

—Rapidez del cambio tecnológico —incluyendo la del sistema de redes-.

—Rapidez del cambio en las tarifas de las comunicaciones, etc.

—Aumento constante del valor de la TIC y de la capacidad computacional.

- Factores internos de la comunidad universitaria y de las instituciones:

—Diferencias en la estructura organizacional.

—Diferencia en los objetivos que se persiguen con la educación abierta y el aprendizaje electrónico.

—Falta de marco de referencia, definiciones y metodología comunes para el análisis de costes.

-Falta de conocimiento claro de los parámetros y condiciones para determinar costes en el campo de la pedagogía y la enseñanza, como por ejemplo, el grado de mediación del instructor en el aprendizaje electrónico, nivel de adaptación a diferentes estilos de aprendizaje, grado de combinación de estilos de aprendizaje.

—Falta de parámetros adecuados que permitan evaluar el cálculo de costes. 
—Responsabilidad, por las iniciativas de aprendizaje electrónico, distribuida en diferentes unidades de la institución.

Además de tener que enfrentar la complejidad del contexto de la educación superior y los desarrollos en metodología de costeo, quienes deseen evaluar los costes del aprendizaje electrónico también se enfrentan al problema de recolectar la información necesaria. Por lo general, la información sobre la carga laboral del cuerpo docente, los costes administrativos compartidos y los costes ocultos e indirectos no se encuentra fácilmente disponible.

Finalmente, la actual dificultad de identificar y estimar los costes asociados con las innovaciones tecnológicas educativas se puede atribuir a la forma en como éstas tienden a aparecer. Los pioneros en enseñanza con tecnología educativa y aprendizaje electrónico por lo general obtienen resultados a costes muy bajos en términos monetarios, aunque el coste en términos de horas trabajadas pueda ser alto; con frecuencia actúan movidos por un interés genuino en la calidad del aprendizaje y la enseñanza, y/o por el entusiasmo informático, sin preocuparse en exceso por los costes.

\section{Coste-eficacia}

En la figura 5 se describe gráficamente la relación coste-eficacia: cinco aproximaciones de EAD se posicionan sobre las coordenadas $x-y$. El caso ideal sería una aproximación de bajo coste y alta eficacia o rendimiento, esto es, la parte superior izquierda del gráfico; por el contrario, el caso de menor valor estaría posicionado en la parte inferior de la derecha: alto coste y bajo rendimiento. Las universidades virtuales son caras, requieren un gran ancho de banda y la necesidad de una infraestructura de TIC, lo que causa el desaliento incluso en naciones desarrolladas. No obstante, hay que tener en cuenta que se está prácticamente en los inicios.

La comparación del coste promedio por estudiante en EAD con algunas instituciones tradicionales próximas muestra que no siempre el mejor ratio se lo llevan las primeras. La tabla I muestra el ratio de eficacia — definido como el coste promedio por estudiante en EAD dividido por el de la institución tradicional- en una serie de proyectos; cuando el ratio es menor que 1 significa que el sistema a distancia es más barato que el presencial; si resulta mayor, implica lo contrario. ¿Qué justificaría esto último? Que se trate del único modo de llegar a algunas audiencias objetivo.

\section{CONCLUYENDO}

Algunos de los aspectos más controvertidos de la EAD serían:

- Por su propia naturaleza y por el perfil de sus alumnos, es más susceptible de abandono por parte de éstos que el caso de la enseñanza presencial.

- Se deben analizar con mucha cautela los muchos estudios de calidad que se pueden presentar, pues no es frecuente que las metodologías de investigación pueden encerrar importantes carencias en su diseño.

- Aparecen con frecuencia acciones formativas de EAD basados en las TIC que no ayudan a la consecución de un mejor aprendizaje. 
- Por otra parte, las TIC, aunque levantan algunas barreras, presentan otras nuevas, como son el analfabetismo informático o la brecha digital entre inforricos e infopobres.

- Por último, las economías de escala no siempre responden de un modo eficaz a los planteamientos económicos de las instituciones.

\section{BIBLIOGRAFÍA}

AREA, M. (2004): Nuevas tecnologías, educación a distancia y la mercantilización de la formación, Revista Iberoamericana de Educación, enero 2004.

CLAEYS C. (2004): Costeo del aprendizaje electrónico, en la página web http://www.columbus-

GARCíA ARETIO, L. (1987): Rendimiento académico y abandono en la educación superior a distancia, Madrid: ICE-UNED.

García ARETIO, L. (1994): Educación a distancia hoy, Colección Educación Permanente, UNED, p. 152.

GAY, L. R. y AIRASIAN, P. (2000): Educational Research: Competencies for Analysis and Application, Merrill/Prentice Hall, pp. 367-411.

MARTíneZ, C. (1986): La práctica tutorial en la Universidad Nacional de Educación a Distancia, Madrid: UNED (citado por García Aretio, 1994).

RUIPÉREZ, G. (2003): Internet y su aplicación a la formación, Conferencia en la Jornadas Técnicas de la Feria de Empleo, Bilbao, noviembre de 2003.

SARTORI, G. (2002): Homo videns, Taurus Pensamiento, 206 págs.

Woodley, A. y Parlett, M. (1983): Student drop-out, Teaching at a Distance, n. 24 (citado por García Aretio, 1994).

YUDICE, F. (2002): El recurso de la cultura. Usos de la cultura en la era global, Barcelona, Gedisa 


\section{TABLA Y FIGURAS}

Tabla I

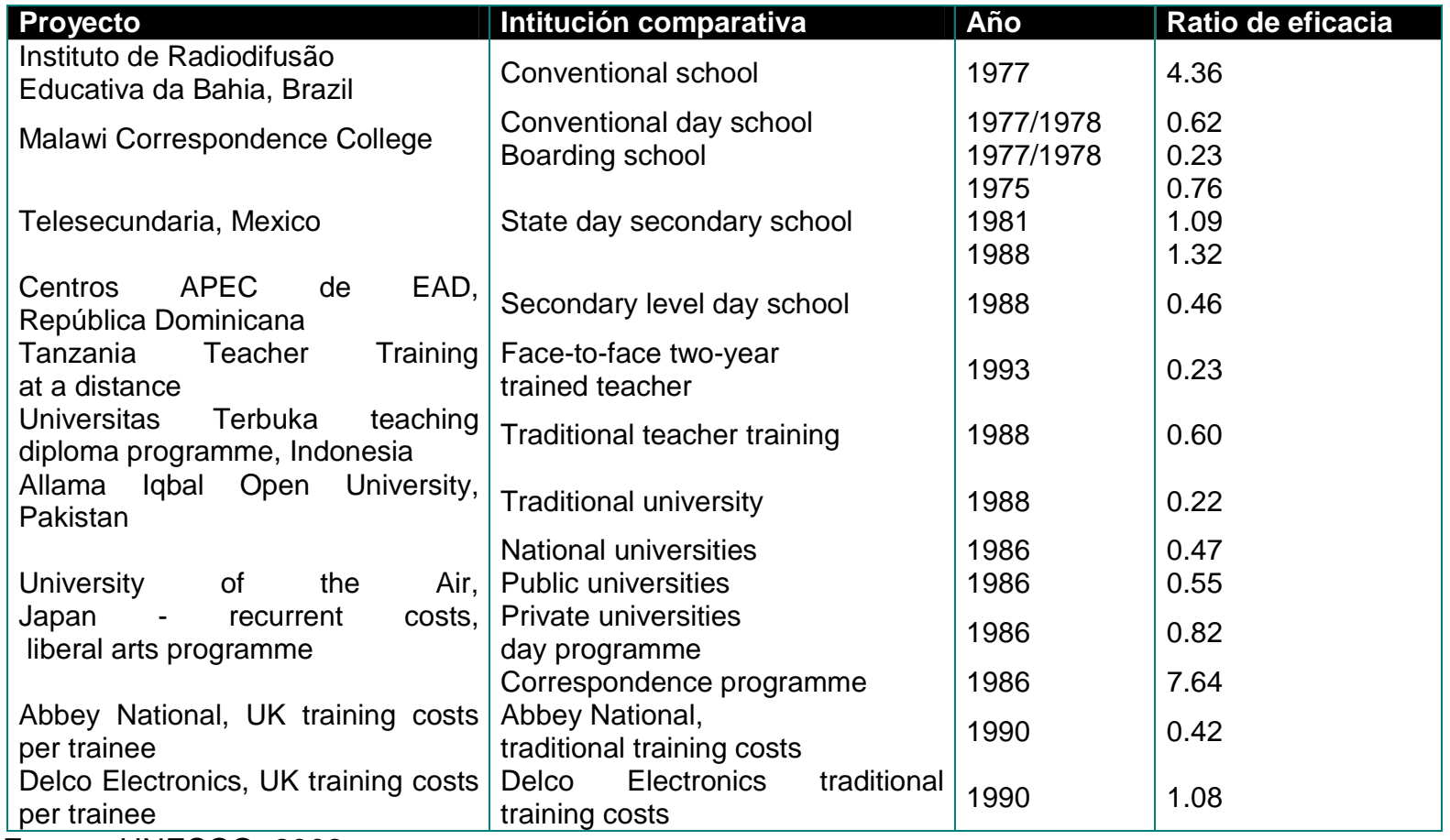

Fuente: UNESCO, 2002

FIGURA 1.

Retención de alumnos en la UNED desde sus comienzos a 1981

1973-1981

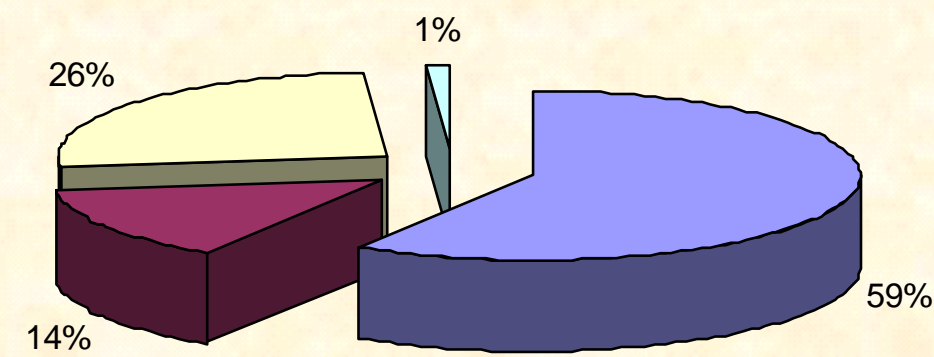

$\square$ abandono sin comienzo $\square$ abandono real $\square$ continuaron $\square$ licenciados

Fuente: García Aretio (1994). 
FIGURA 2.

Las principales causas de abandono en los alumnos de la UNED

Causas de abandono

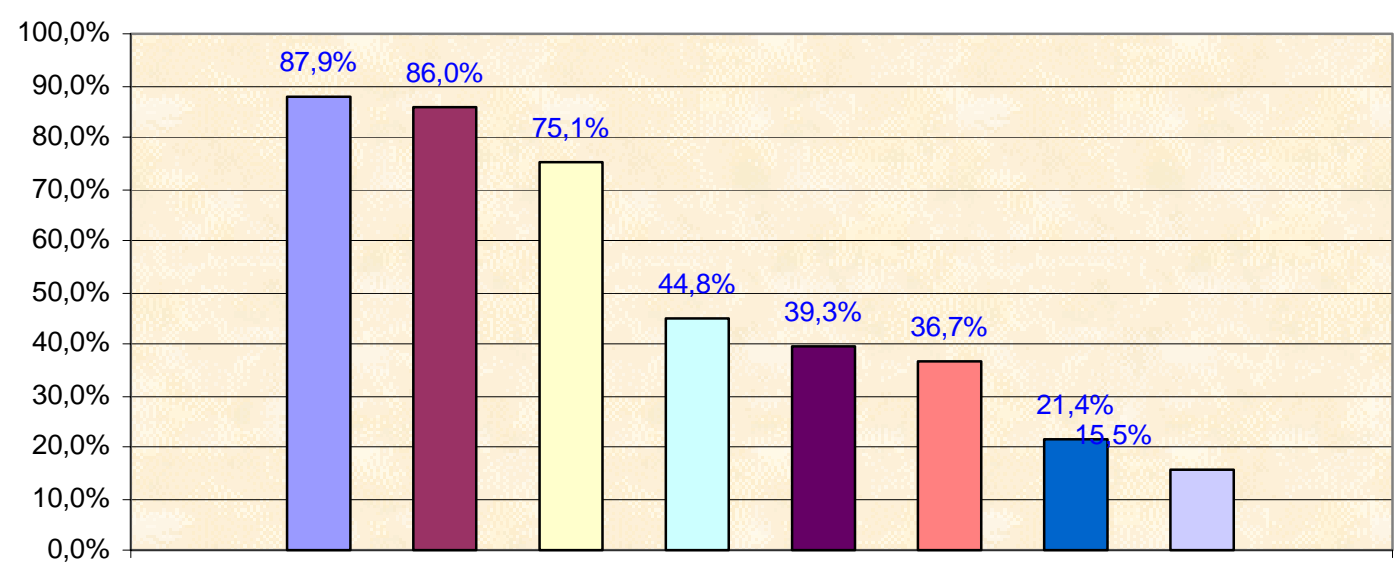

\begin{tabular}{|l}
\hline Falta de tiempo para estudiar \\
$\square$ Dificultades de estudio independiente \\
$\square$ Falta de preparación previa \\
$\square$ Materiales didácticos deficientes \\
$\square$ Falta de comunicación entre el profesor Sede Central y los alumnos \\
$\square$ Devolución tardía y poco clara de los resultados en su aprendizaje \\
$\square$ Falta de comunicación entre profesor-tutor y alumno \\
$\square$ Formación deficiente del profesor-tutor
\end{tabular}

Fuente: García Aretio (1994).

FIGURA 3.

Medios educacionales subsumidos por la web

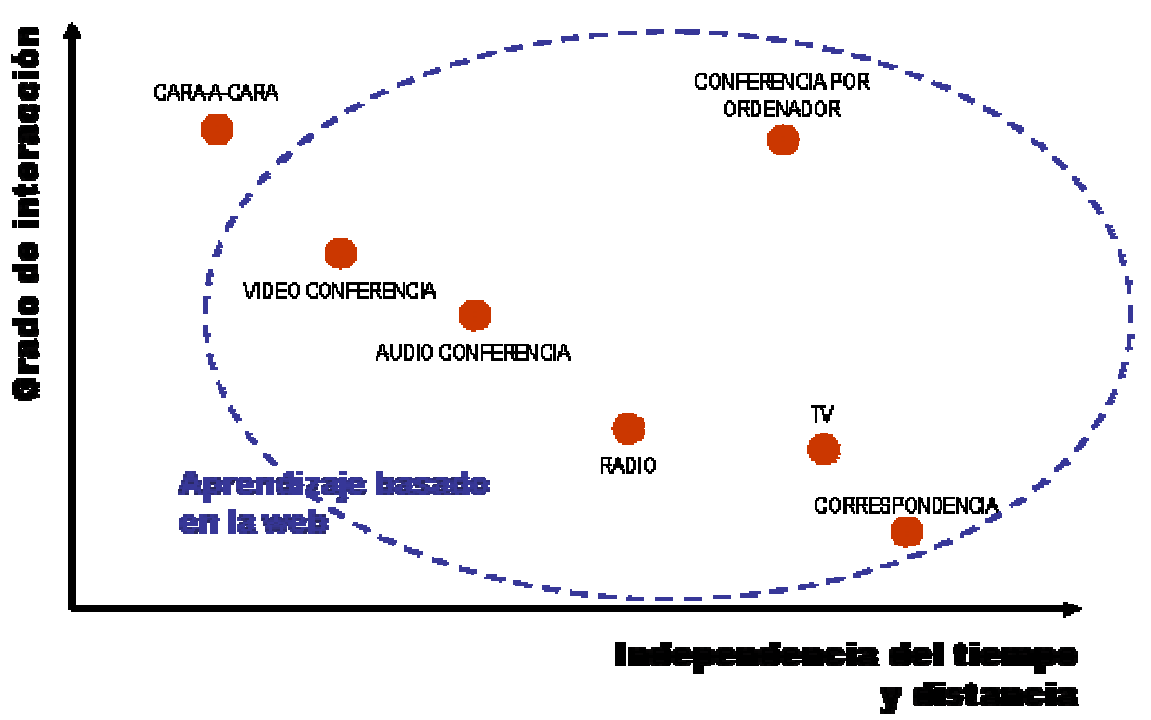


FIGURA 4.

Usuarios de Internet por regiones mundiales.

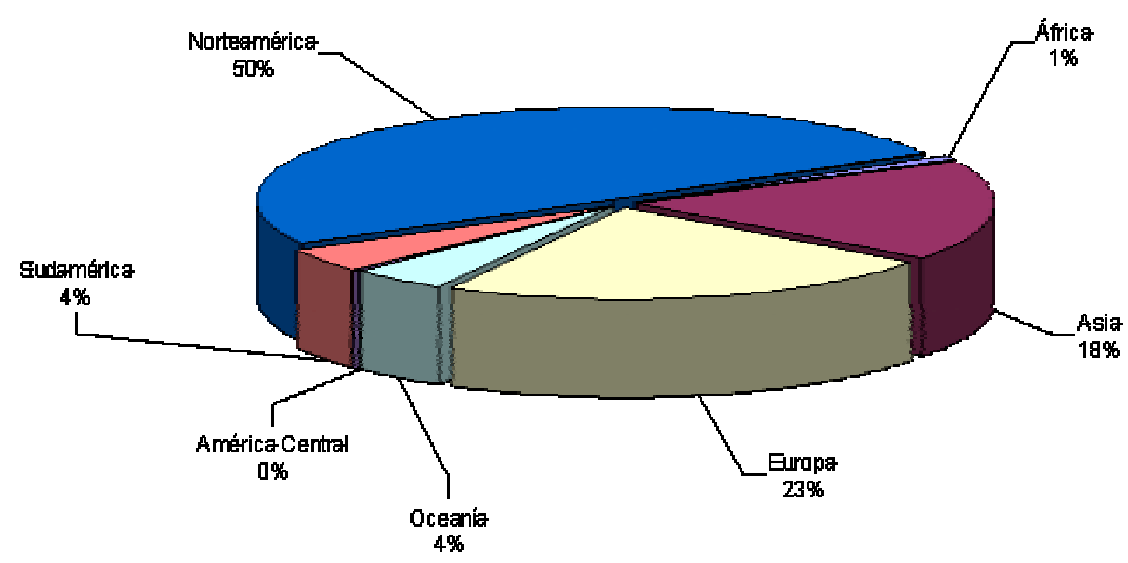

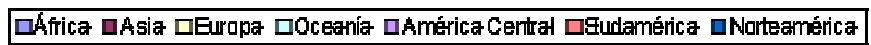

Fuente: Telecordia Internet Sizer (2001).

FIGURA 5.

Relación coste-eficacia para distintas modalidades de EAD;

la flecha indica el avance en el tiempo.

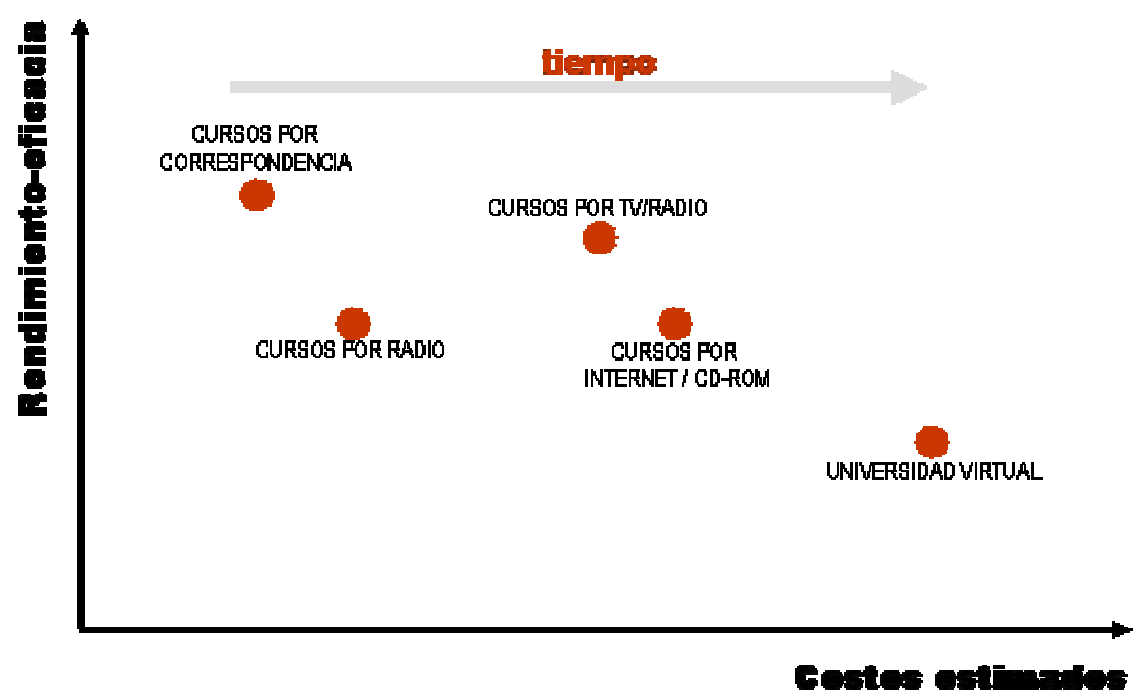




\title{
Contactar
}

Revista lberoamericana de Educación

\author{
Principal OEI
}

